\title{
Hygrothermal behaviour of flax shives used as insulation material in an attic of a traditional house
}

\author{
Franck Brachelet ${ }^{1, *}$, Mounir Asli ${ }^{1}$, Emmanuel Antczak ${ }^{1}$, Flore Brue $^{2}$, Didier Defer ${ }^{1}$, and Alain Lucas $^{3}$ \\ ${ }^{1}$ Univ. Artois, EA 4515, Laboratoire de Génie Civil et géo-Environnement, F-62400 Béthune, France \\ ${ }^{2}$ UC Lille, Yncréa HdF - HEI, Laboratoire de Génie Civil et géo-Environnement, 13 rue de Toul, F-59046 Lille, France \\ ${ }^{3} \mathrm{~cd} 2 \mathrm{e}$, Base du 11/19, F-62750 Loos en Gohelle, France
}

\begin{abstract}
Western Europe area is one of the world regions where traditionally flax is grown. Whether in France or Belgium, flax is mainly used for the production of fabrics. When separating the different components of the plant, small woody elements called shives which represented about $50 \%$ of the production, are recovered. This work aims to study the behavior of flax shives used as insulation material in an attic of a French Flanders traditional house. Main scientific issues related to the study of heat and mass transfer is to determine the evolution of the thermal characteristics of the material and its durability. First, we propose a laboratory analysis of the hygrothermal behavior of shives by conventional thermal methods for characterizing thermal conductivity and volumetric heat (heat flux method), sorption curve is also determined. Then we focus on an experimental site which is an independent house where the old insulation of the attic in glass wool was replaced by flax shives. The hygrothermal behavior of this new insulation was monitored with humidity and temperature sensors for ten months. The thermal performances determined for a raw form of the shives in the laboratory are encouraging and hygrometric results show advantageous behavior whatever the season.
\end{abstract}

\section{Introduction}

Production areas of flax cultivating essentially concern the Western part of Europe because flax does not like heat and deficiency of water. Flax shives are small fragments resulting from the separation of the flax fibers from their stems. Currently, shives are used for animals bedding or are integrated into the component mixture of particle boards. Some researches on materials focus on flax as additional fiber for cementitious materials. In the same way as the straw in the eco-construction, project partners wish to valorize flax shives as a bio-based insulation material. Moreover, from an economic point of view, a circular economy approach could be an alternative solution in the global energy context $[1,2,3]$. The principle is to valorize local natural resources and to use them within the framework of short circuits of distribution [4]. In this project, we want to use the product close to its raw form, the least processed as possible in order to reduce energy consumption. So, flax shives have only been treated with sodium carbonate and bicarbonate to accentuate the fire-resistant property and stop the appearance of insects. The first part of this work is devoted to the thermal characterization of shives in laboratory conditions and the second concerns the monitoring of the hygrothermal behavior of the flax shives used as insulation material in the attic of a traditional house.

\section{Thermal Characterization}

\subsection{Density as a function of settlement}

The protocol consists in weighing a cylindrical test piece of $16 \times 32$, filled with more or less packed flax shives in the laboratory environment at a relative humidity of $50 \%$. For the lowest density (E1), it is poured freely into the sample holder, and then, in order to increase its density, it is placed in layers by means of a mass (E2, E4, E8). The number indicated specifies how many layers are produced. Each measurement is performed 3 times, only the average value is showed in figure 1 .

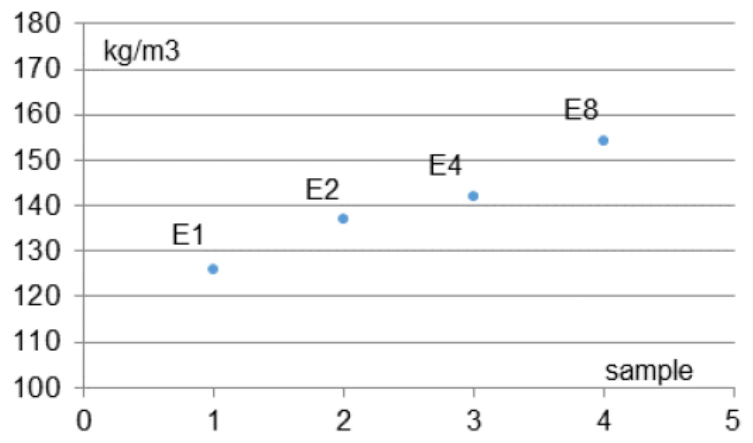

Fig. 1. Densities obtained for flax shives.

\footnotetext{
Corresponding author: author@e-mail.org
} 
The densities obtained are quite high compared to those found for traditional insulation. It ranges between 126 $\mathrm{kg} / \mathrm{m} 3$ and $154 \mathrm{~kg} / \mathrm{m} 3$, with an average value of 140 $\mathrm{kg} / \mathrm{m} 3$. Compared to existing products for this type of insulation, bulk mineral wools have a density between $20 \mathrm{~kg} / \mathrm{m} 3$ and $80 \mathrm{~kg} / \mathrm{m} 3$, the cellulose wadding is blown to a density of $50 \mathrm{~kg} / \mathrm{m} 3$.

\subsection{Thermal conductivity}

Thermal characteristics are measured in steady state with the heat flow meter method according to the standard [5]. The guarded hot plate aims to measure thermal conductivity and specific heat of the tested material. The experimental setup consists on maintaining a temperature difference $(\Delta \mathrm{T})$ between two parallel flat plates. A sample with a known thickness (e) is placed between them. Thermal Resistance $R\left[\mathrm{~m}^{2} . \mathrm{K} / \mathrm{W}\right]$ is calculated as follow:

$$
R=\Delta T / \Phi
$$

Where $\Phi\left(\mathrm{W} . \mathrm{m}^{-2}\right)$ is the average of the measured heat flux. Thermal conductivity is deduced from the measurement of the thickness (e).

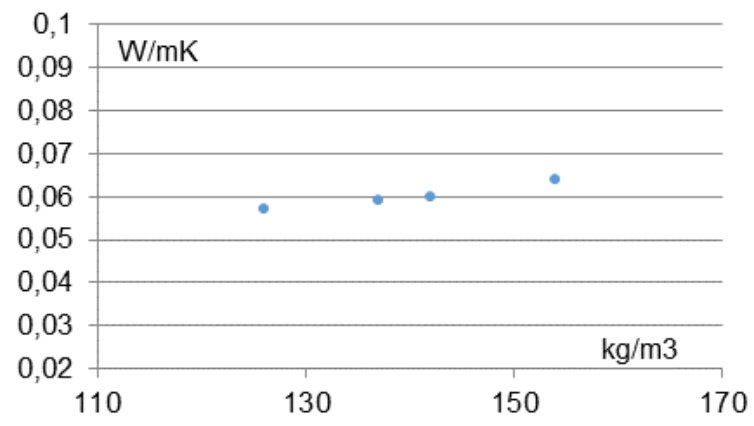

Fig. 2. Thermal conductivity of flax shives versus density.

One can notice that an increase of the thermal conductivity is correlated with the density. It ranges between $0.057 \mathrm{~W} \cdot \mathrm{m}^{-1} \mathrm{~K}^{-1}$ and $0.064 \mathrm{~W} \cdot \mathrm{m}^{-1} \mathrm{~K}^{-1}$. It is a fairly high value compared to a traditional insulation which is around $0.04 \mathrm{~W} \cdot \mathrm{m}^{-1} \mathrm{~K}^{-1}$. Measurements have also been made for moisture. Indeed, under in situ conditions of use, the relative humidity of the environment varies greatly according to the seasons in the volume of the attic.

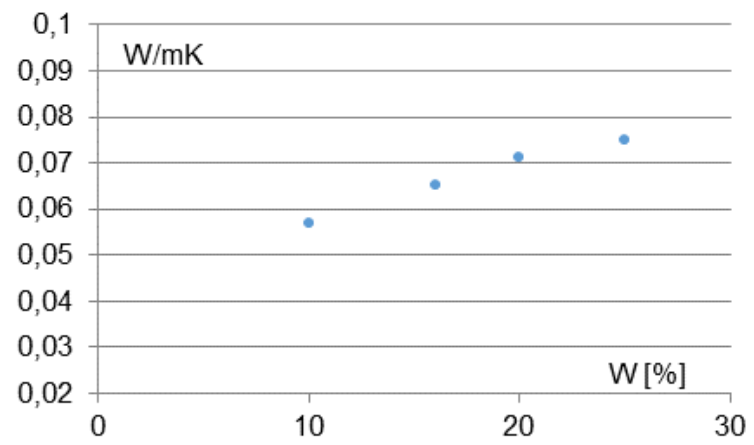

Fig. 3. Thermal conductivity versus humidity.
Fig. 3 shows the evolution of the thermal conductivity as a function of the mass water content $\mathrm{W}(10 \%, 16 \%$, $20 \%, 25 \%$ ) which correspond to a relative humidity ranging from $50 \%$ to $95 \%$ for the environment, close to the in situ use conditions of the material use. The measurements were made for a density at $\mathrm{RH}=50 \%$ of $130 \mathrm{~kg} / \mathrm{m}^{3}$, this corresponds to the material during its implementation and the first months of its use. The thermal conductivity varies from $0.057 \mathrm{~W} \cdot \mathrm{m}^{-1} \cdot \mathrm{K}^{-1}$ to $0.075 \mathrm{~W} \cdot \mathrm{m}^{-1} \cdot \mathrm{K}^{-1}$, an increase of about $30 \%$. The thermal resistances obtained vary from $4 \mathrm{~m}^{2} . \mathrm{K}^{-\mathrm{W}^{-1}}$ to 5.25 $\mathrm{m}^{2} . \mathrm{K} . \mathrm{W}^{-1}$ which corresponds to a thickness of mineral wool of $20 \mathrm{~cm}$, perfectly laid.

\subsection{Specific heat}

It is evaluated with the same device, only the applied thermal loads changes. First the previous system (sample and heat flux meter) is submitted to a constant temperature until reaching the steady-state, then we change towards another constant temperature and wait for the steady-state again. We assume that the sample goes from initial time $t_{\text {ini }}$ (at temperature $T_{\text {ini }}$ ) to final time $t_{\text {fin. }}$. (at temperature $T_{\text {fin }}$ ), between these instances the sample stores an amount of energy Q $\left(\mathrm{J}_{\mathrm{m}} \mathrm{m}^{-2}\right)$. In this case the heat capacity $\mathrm{C}\left(\mathrm{J} \cdot \mathrm{K}^{-1} \cdot \mathrm{m}^{-2}\right)$ is calculated as follows:

$$
\begin{gathered}
Q=\int_{\text {tini }}^{t f i n} \Delta \Phi \cdot d t \\
\mathrm{C}=\mathrm{Q} / \Delta \mathrm{T}
\end{gathered}
$$

Where $\Delta \mathrm{T}=\mathrm{T}_{\text {fin }}-\mathrm{T}_{\text {ini }}$

The specific heat $\mathrm{c}\left[\mathrm{J} \cdot \mathrm{K}^{-1} \cdot \mathrm{kg}^{-1}\right]$ is calculated:

$$
\mathrm{C}=\mathrm{c} /(\text { p.A.e) }
$$

Where $\rho$ [kg.m-3] and e [m] are the material density and thickness respectively, concerning the heat flux density measured we assume that $A=1 \mathrm{~m}^{2}$.

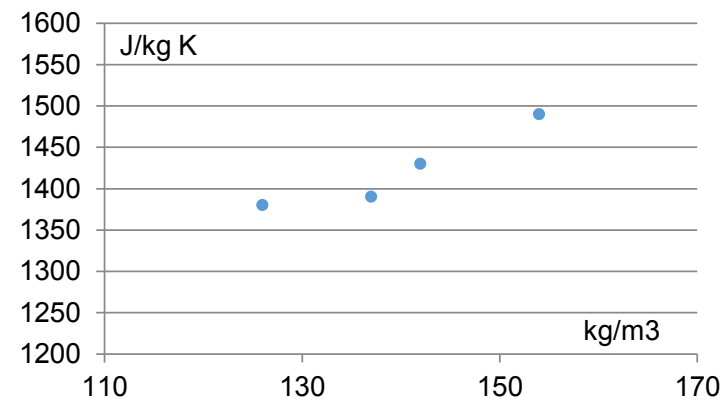

Fig. 4. Specific heat of flax shives.

The values obtained are in the range usually observed for insulating materials, between $1200 \mathrm{~J}^{\mathrm{kg}} \mathrm{kg}^{-1} \mathrm{~K}^{-1}$ and 1600 $\mathrm{J}_{\mathrm{kg}} \mathrm{k}^{-1} \mathrm{~K}^{-1}$. The interesting point is that the flax shives have a density higher than those of the traditional insulation.

\subsection{Sorption curve}


The use of a bio-based material requires knowing its capacity to regulate the ambient humidity. The measurement protocol consists of placing the sample in a climate chamber controlled at constant temperature and imposing relative humidity ranges from $0 \%$ to $95 \%$ (depending on the performance of the enclosure) [6].

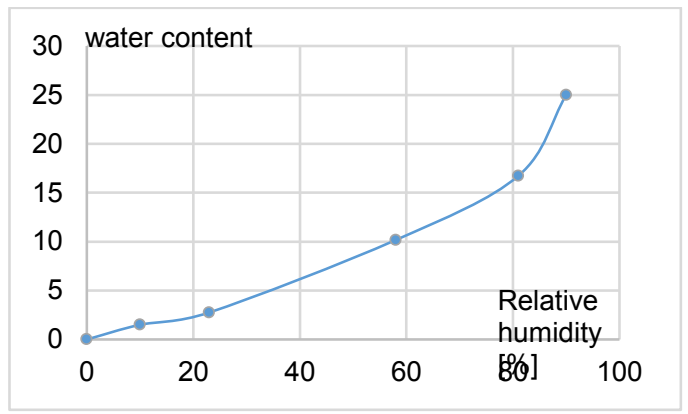

Fig.5. Sorption curve of flax shives.

Figure 5 represents the sorption curve which does not reveal a true level between $\mathrm{RH}=10 \%$ and $\mathrm{RH}=60 \%$ as can usually be observed. This may be due to the natural presence of fines and sodium bicarbonate.

\section{Instrumentation set up and monitoring}

The experimentation set up concerns a typical housing of the French maritime Flanders. The materials used are traditionally brick and cob, the construction dates back to 1610 and is made on a single level of ground floor. The aim of this experimentation is to quantify the hygrothermal properties of flax shives used in its bulk form as thermal insulation. This house is and will stay occupied during the experimentation. Sensors record evolutions of temperature and relative humidity before, during and after the implementation of flax shives. However, the use in bulk form of flax shives need to find a non-occupied room for the thermal rehabilitation, that's why the experimentation is made in the attic of the house. The attic is $75 \mathrm{~m}^{2}$ and presents recent roof and vapor barrier. Before thermal rehabilitation, the attic was isolated with glass wool since several years. Thus the glass wool was compacted with time and has given dust and microfibers in the attic. Figure 6 presents the attic after the thermal rehabilitation.

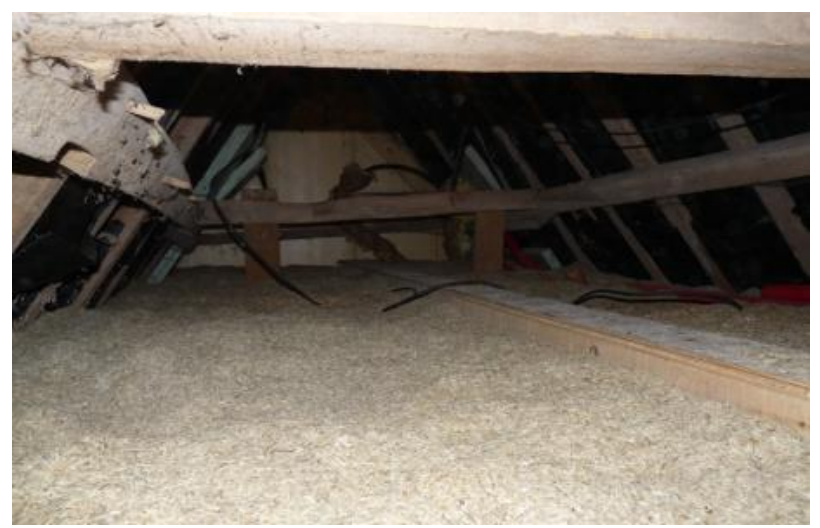

Fig.6. Attic after thermal rehabilitation with flax shives (thickness $30 \mathrm{~cm}$ ).

The installation of an instrumentation aims to monitor the hygrothermal behavior of the material, depending on the in situ conditions and according to the seasons. It must be ensured that the thermal performances are sufficient and that the regulation of the humidity is effective. The sensors used to monitor the humidity and the temperature evolutions are thermohygrometers (HMP 110 Vaisala and recorders Easylog EL-USB 2+) and thermocouples. The measurements of temperature and of hygrometry are carried out in the ambient volumes and in the mass of the sample. A support (Figure 7), vertically positioned, consisting of wooden frame and PVC mesh allows the thermocouples and thermohygrometers to be placed at equal distances within the thickness of the material. The use of a support with large mesh makes it possible not to disturb the arrangement of the flax shives nor transfers of moisture and heat.

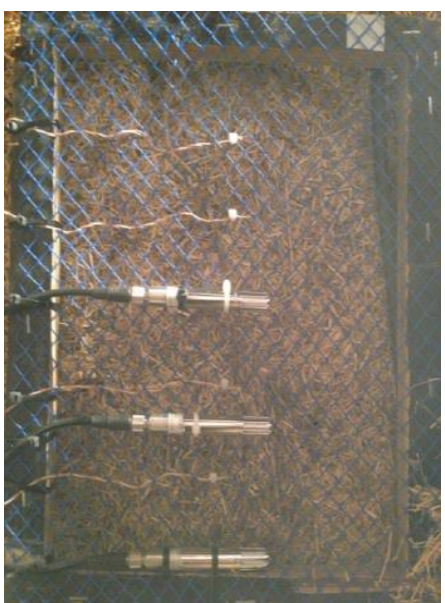

Fig.7. Positioning of probes on the support.

Measurements of ambient humidity in the attic and in the inhabited part of the house are also carried out, they make it possible to demonstrate the influence of the behavior of the flax shives on the ambient volumes. The sensors are connected to a datalogger which records data every $20 \mathrm{~min}$. The distribution of the sensors is done according to the following sketch:

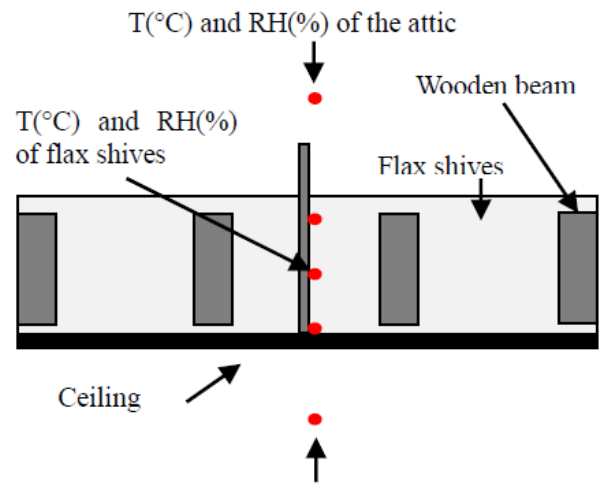

$\mathrm{T}\left({ }^{\circ} \mathrm{C}\right)$ and $\mathrm{RH}(\%)$ of the inhabited volume 
Fig.8. Positioning of probes in the house.

\section{In situ measurement}

We will first analyze the temperature and humidity data from February to November 2016. To facilitate readability, we selected the months of February and August, representative of the winter and summer periods.

\subsection{February 2016}

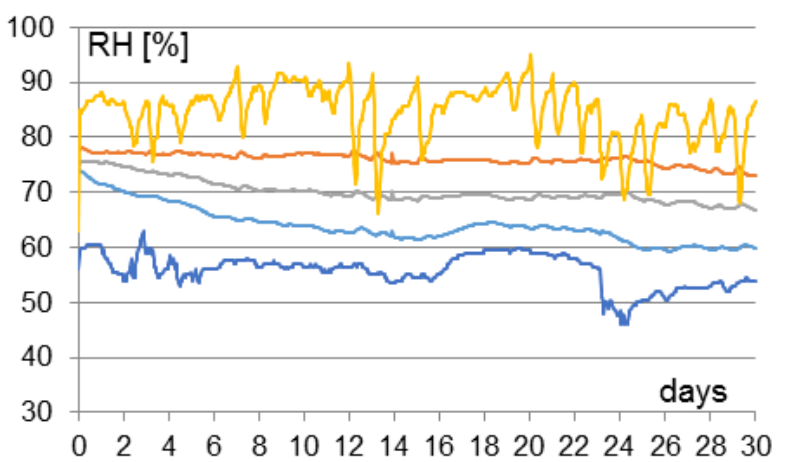

Fig.9. Relative humidity measurements.

The curves shown in the Fig.9 correspond, respectively, from top to bottom, to the attic, to the three measurement levels in the flax shives and to the heated interior volume of the house. As hot air can contain more moisture than cold air, so it is logical to find the heated volume between $\mathrm{RH}=50 \%$ and $\mathrm{RH}=60 \%$. The flax shives in contact with the ceiling seem to be influenced by the volume heated. The relatively high temperature of the flax shives compared to that of the attic means that the relative humidity remains stable despite the variations in the atmosphere of the attic. The values are also influenced by the introduction of wet flax shives at the beginning of measurements.

Curves in Figure 10 show, in decreasing order of temperature, the temperatures of the inhabited volume, then the three sensors placed in the flax shives and at the lowest temperatures, the ambient temperature of the attic. The flax shives fulfill their role well since an average temperature of $10^{\circ} \mathrm{C}$ is observed between the lowest and the highest temperatures, Amplitudes are well attenuated by the thermal insulation.

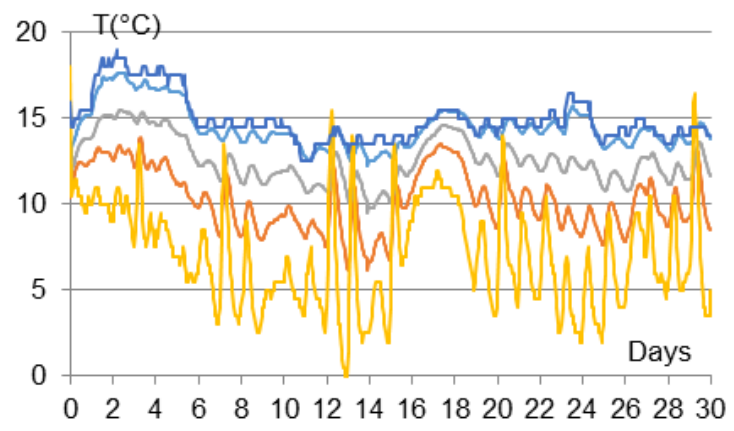

Fig.10. Temperature measurements.

The curves shown in the Fig. 11 correspond, respectively, from top to bottom, to the attic, to the three measurement levels in the flax shives and to the heated interior volume of the house. As hot air can contain more moisture than cold air, so it is logical to find the heated volume between $\mathrm{RH}=50 \%$ and $\mathrm{RH}=60 \%$. The flax shives in contact with the ceiling seem to be influenced by the volume heated. The relatively high temperature of the flax shives compared to that of the attic means that the relative humidity remains stable despite the variations in the atmosphere of the attic. The values are also influenced by the introduction of wet flax shives at the beginning of measurements.

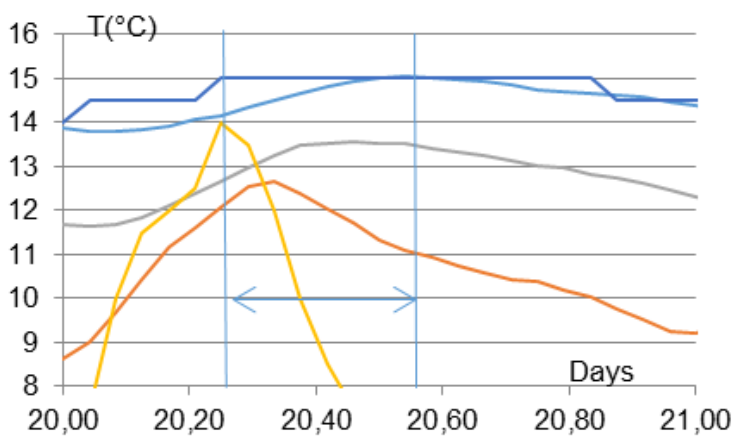

Fig.11. Phase delay.

Curves in Figure 11 show, in decreasing order of temperature, the temperatures of the inhabited volume, then the three sensors placed in the flax shives and at the lowest temperatures, the ambient temperature of the attic. The flax shives fulfill their role well since an average temperature of $10^{\circ} \mathrm{C}$ is observed between the lowest and the highest temperatures, Amplitudes are well attenuated by the thermal insulation.

\subsection{August 2016}

Temperatures are higher in summer hence relative humidity levels are lower at equal amount of moisture in the attic (Figure 13).

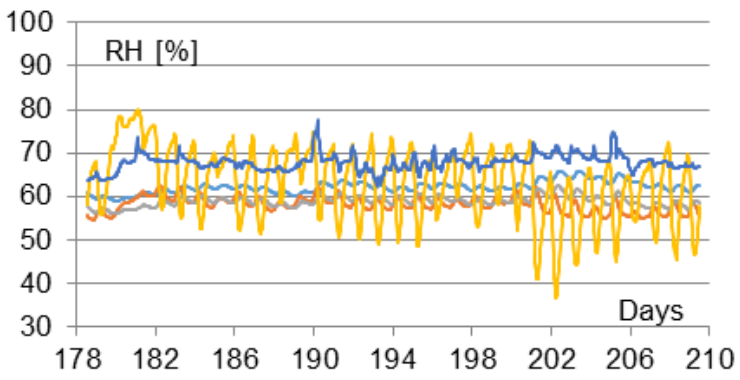

Fig.12. Relative humidity measurements.

Curves become narrower, the average hygrometry of the attic is lower than that of the inhabited volume where human activity produces steam. The relative humidity levels in the flax shives are homogeneous and are around a value of $60 \%$, which is very interesting compared to the use of the wet material a few months previously. 
There are large variations in the amplitudes of the ambient temperature of the attic (up to $21^{\circ} \mathrm{C}$ over $24 \mathrm{H}$ ), which is linked to the summer climatic conditions and the low ventilation of the volume. Amplitudes are well damped since the amplitude variations over $24 \mathrm{H}$ the highest do not exceed $3{ }^{\circ} \mathrm{C}$ in the lower part of the flax shives (Figure 14). The impact on the interior volume is reduced. The characteristics of the materials evolving, the phase delay in summer is $7 \mathrm{H}$ (Figure 15).

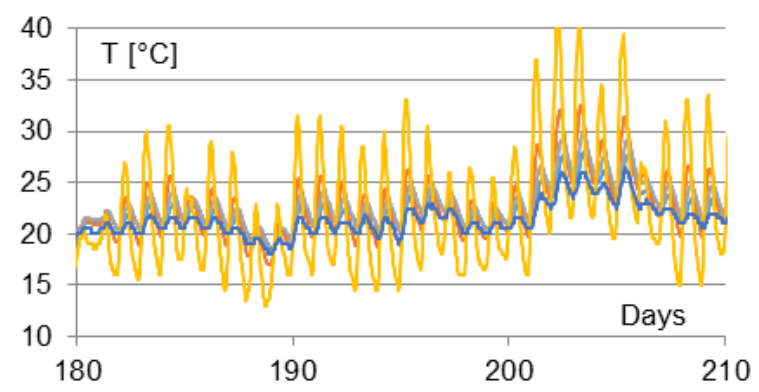

Fig.13. Temperature measurements.

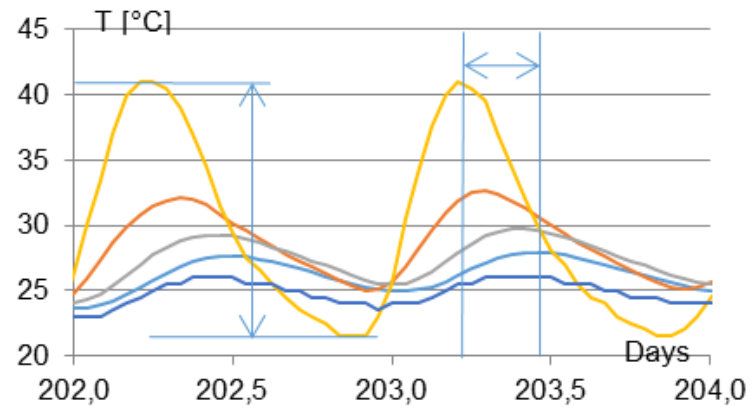

Fig.14. Phase delay.

\section{Conclusions}

The project "Researchers-Citizens" aims at bringing together university laboratories and the regional population in the framework of a participatory action. Our goal is to valorize a material produced locally and to benefit the people of the territory for the renovation of their housing. The flax shives were used in their raw form, harvested on the spot and adjuvanted with a fire retardant used in organic food. The thermal performances determined in the laboratory are encouraging, knowing that for this type of material, the bulk form does not limit the thickness used, it is therefore always possible to obtain an interesting thermal resistance. The material is highlighted thanks to its high density, which favors its inertia and its use in bulk which makes it possible to obtain a layer more airtight than a product in the form of wool, and less sensitive to settlement. Hygrometric readings show advantageous behavior, whatever the season, to fluctuations in relative humidity. The levels found within the material do not indicate any risk of mold growth. The natural and farming origin of the product do not favor the presence of rodents, as these cannot nest because of the impossibility of creating galleries. Flax shives clearly show hygrothermal qualities in the context of a use as bulk insulation for the renovation of traditional buildings. Their low energy cost to their production is obvious, since it is a by-product derived directly from farming.

\section{Acknowledgments}

The "Hauts de France" region is funding the project within the framework of the "Citizen Researchers" call projects.

\section{References}

1. K. Biswas, S.S. Shrestha, M.S. Bhandari, A.O. Desjarlais, Insulation materials for commercial buildings in North America: An assessment of lifetime energy and environmental impacts, Energy Build., vol. 112, pp. 256-269, 1 (2016).

2. K. E. Thomsen, Energy consumption and indoor climate in a residential building before and after comprehensive energy retrofitting, Energy Build., vol. 123, pp. 8-16, 7 (2016).

3. T. Khadiran, M. Z. Hussein, Z. Zainal, R. Rusli, Advanced energy storage materials for building applications and their thermal performance characterization: A review, Renew. Sustain. Energy Rev., vol. 57, pp. 916-928, 5 (2016).

4. S. Lasvaux, F. Achim, P. Garat, B. Peuportier, J. Chevalier, G. HabertT, Correlations in Life Cycle Impact Assessment methods (LCIA) and indicators for construction materials: What matters? Ecol. Indic., vol. 67, pp. 174-182, 8 (2016).

5. NF EN 12664, Thermal performance of building materials and products. Determination of thermal resistance by means of guarded hot plate and heat flow meter methods. Dry and moist products of medium and low thermal resistance, pp. 75-225, (2001).

6. NF EN ISO 12571, Hygrothermal performance of building materials and products- Determination of hygroscopic sorption properties, (2013).

5. R.M. Kozasowski, M. Mackiewicz-Talarczyk, A.M. Allam, Handbook of Natural Fibres, Pages, pp. 56113, (2012).

6. D. Lelievre, T. Colinart, P. Glouannec, Hygrothermal behavior of bio-based building materials including hysteresis effects: Experimental and numerical analyses, Energy Build., vol. 84, pp. 617-627, 12 (2014).

7. E. Sassine, Z. Younsi, Y. Cherif, E. Antczak, Frequency domain regression method to predict thermal behavior of brick wall of existing buildings, Applied Thermal Engineering, vol. 114, Pages 2435, 3 (2017). 
8. O. Vololonirina, M. Coutand, B. Perrin, Characterization of hygrothermal properties of wood-based products - Impact of moisture content and temperature, Constr. Build. Mater., vol. 63, pp. 223-233, 9 (2014). 